\title{
Lie Algebras and Suppression of Decoherence in Open Quantum Systems
}

\author{
William Gordon Ritter \\ Harvard University Department of Physics \\ 17 Oxford St., Cambridge, MA 02138
}

(Dated: April 15, 2005)

\begin{abstract}
Since there are many examples in which no decoherence-free subsystems exist (among them all cases where the error generators act irreducibly on the system Hilbert space), it is of interest to search for novel mechanisms which suppress decoherence in these more general cases. Drawing on recent work (quant-ph/0502153) we present three results which indicate decoherence suppression without the need for noiseless subsystems. There is a certain trade-off; our results do not necessarily apply to an arbitrary initial density matrix, or for completely generic noise parameters. On the other hand, our computational methods are novel and the result - suppression of decoherence in the error-algebra approach without noiseless subsystems - is an interesting new direction.
\end{abstract}

PACS numbers: 03.65.Yz, 03.67.Pp, 03.65.Fd, 02.20.Qs, 02.20.Sv

Keywords: decoherence, quantum channels, open systems, Markovian dynamics

\section{INTRODUCTION}

The central obstacle in the experimental realization of quantum computers has proven to be maintaining the quantum coherence of states 1]. The main cause of this degradation of the quantum coherence is the coupling of the computer to the environment, and the decoherence induced by this coupling.

A variety of schemes for protecting quantum information have been developed, including quantum error correction codes [2, 3, 4, 5], decoherence free subspaces 6. 7, 8, 9, 10], noiseless subsystems [11], bang-bang decoupling 12], and topological quantum computation [13]. The first four of these techniques are closely related to each other and can be described in a simple unified framework based on representations of the algebra of errors 11, 14, 15]. More recently, Zanardi and Lloyd 16. showed that topological quantum computation also falls into the error-algebra framework.

Our approach, like those mentioned above, uses the (Lie algebraic) structure of the physical decoherence process itself to protect quantum coherence. We start from the assumption that the Lindblad operators generate a representation of a Lie algebra, and show that if this representation is irreducible, then decoherence-free subsystems and subspaces will not exist. Thus if one is to have any hope of controlling decoherence in the irreducible case, some other mechanism is needed.

\section{A. Markovian Dynamics}

The dynamics of a quantum system $A$ coupled to a heat bath $B$, which together form a closed system, is described by a Hamiltonian [17]:

$$
\left(H_{A} \otimes \mathbf{1}_{B}\right)+\left(\mathbf{1}_{A} \otimes H_{B}\right)+H_{I},
$$

where $H_{A}, H_{B}$, and $H_{I}$ are the system, bath, and interaction Hamiltonians respectively. The Markovian Master equation [18, 19] (also "semigroup master equation" or
SME) provides the most general form for time evolution of the system density operator $\rho=\rho_{A}$, which acts on a Hilbert space $\mathcal{H}_{A}$ :

$$
\frac{d \rho}{d t}=-\frac{i}{\hbar}\left[H_{A}, \rho\right]+\mathcal{L}(\rho) .
$$

Here $\mathcal{L}$ is called the Lindbladian or dissipator, and takes the general form

$$
\begin{aligned}
\mathcal{L}(\rho) & =\frac{1}{2} \sum_{\alpha, \beta} a_{\alpha, \beta} L_{F_{\alpha}, F_{\beta}}(\rho), \\
L_{F_{\alpha}, F_{\beta}}(\rho) & \equiv\left[F_{\alpha}, \rho F_{\beta}^{\dagger}\right]+\left[F_{\alpha} \rho, F_{\beta}^{\dagger}\right] .
\end{aligned}
$$

The equation of motion remains invariant under an arbitrary unitary transformation of the Lindblad operators. The special case corresponding to $\mathcal{L}(\rho)=0$ is the von Neumann equation, and describes unitary evolution. Therefore in general, $\mathcal{L}(\rho)$ contains precisely those terms in the evolution equation which are responsible for decoherence. The derivation of eqns. (2) and (3) from fundamental assumptions is done in many places; Bacon et al. [20] have a particularly nice treatment.

The coefficient matrix $\left(a_{\alpha, \beta}\right)$ is assumed to be timeindependent and Hermitian. By diagonalizing $a$, it follows that (2) may also be written in "diagonal standard form," replacing the $F_{\alpha}$ operators by suitable linear combinations $V_{a}$. This yields

$$
\mathcal{L}(\rho)=\frac{1}{2} \sum_{a} L_{V_{a}, V_{a}}(\rho) .
$$

The noise coefficients $a_{\alpha, \beta}$ have been absorbed within the operators $V_{a}$. From (4), using the relation $L_{A, A}=[A,[\rho, A]]$ which holds for a Hermitian operator $A$, we find

$$
\begin{aligned}
\mathcal{L}(\rho) & =\frac{1}{2} \sum_{a}\left[V_{a},\left[\rho, V_{a}\right]\right]=\sum_{a}\left(V_{a} \rho V_{a}-\frac{1}{2}\left\{\rho, V_{a}^{2}\right\}\right) \\
& =\sum_{a} V_{a} \rho V_{a}-\frac{1}{2}\left\{\rho, \sum_{a} V_{a}^{2}\right\} .
\end{aligned}
$$


If $\sum_{a} V_{a}^{2}=c \mathbf{1}$ where $c \in \mathbb{R}$ is a constant, then we have

$$
\mathcal{L}(\rho)=\sum_{a} V_{a} \rho V_{a}-c \rho .
$$

The first term is reminiscent of a quantum channel with Hermitian Kraus operators. Indeed, an equivalent form of the Markov master equation is

$$
\frac{d}{d t} \rho_{t}=-i\left[H, \rho_{t}\right]+\Phi\left[\rho_{t}\right]-\frac{1}{2}\left\{\Phi^{*}(\mathbf{1}), \rho_{t}\right\}
$$

where $\Phi$ is a completely positive map [21, p. 18, II.5.5].

In applications to real-world systems, the matrix elements $a_{\alpha \beta}$ contain physical parameters such as lifetimes, longitudinal or transverse relaxation times, stationary magnetization, etc. We will show that particularly simple dynamics emerge if the coefficient matrix is not only Hermitian but also real (hence symmetric) or approximately so.

The $F_{\alpha}$ describe various decoherence processes, and for this reason, they are often called error generators. The $F_{\alpha}$ are determined implicitly by the interaction Hamiltonian

$$
H_{I}=\sum_{\alpha} F_{\alpha} \otimes B_{\alpha},
$$

where $\left\{B_{\alpha}\right\}$ is a collection of operators on $\mathcal{H}_{B}$, called the heat bath operators. Let $\mathfrak{g l}\left(\mathcal{H}_{A}\right)$ denote the vector space of all linear transformations on $\mathcal{H}_{A}$, not necessarily invertible. This is the Lie algebra of the group $G L(n)$ where $n=\operatorname{dim}\left(\mathcal{H}_{A}\right)$. We now state the main assumption of the paper.

Assumption 1. There exists a Lie algebra $\mathfrak{g}$ and a faithful representation $\phi: \mathfrak{g} \rightarrow \mathfrak{g l}\left(\mathcal{H}_{A}\right)$ such that

$$
F_{\alpha}=\phi\left(f_{\alpha}\right), \quad \alpha=0 \ldots M
$$

for some linearly independent set $\left\{f_{\alpha}\right\} \subset \mathfrak{g}$.

This assumption is also basic to many other studies of decoherence; see [9, 20, 22] for a few. The trace of any generator of any representation of a compact simple Lie algebra is zero [23, Thm. 8.9]. Further, we are primarily interested in representations for which all the generators can be chosen to be Hermitian. This is always possible for compact Lie algebras [23, Sec. 2.4]. We may therefore take $F_{\alpha}$ to be traceless and Hermitian.

Decoherence processes in which the $F_{\alpha}$ are Hermitian are mathematically much simpler than the most general process, and this is often the case of interest to applications in quantum computing. We recall for clarity the standard example of a quantum computer made of $K$ qubits with $n=2^{K}$ dimensional register Hilbert space.

Each qubit has four possibilities: no error, or an error generated by one of the three Pauli matrices. This means that each qubit independently undergoes the action of the standard depolarizing channel

$$
\rho \rightarrow \sum_{\mu} M_{\mu} \rho M_{\mu}
$$

with Kraus operators

$$
M_{0}=\sqrt{1-p} \mathbf{1}, \quad M_{i}=\sqrt{\frac{p}{Z}} \sigma_{i} \quad(i=1 \ldots 3) .
$$

where $\sigma_{i}$ are Pauli matrices and $Z$ is a normalization constant equal to $3 / 4$ for the spin- $1 / 2$ representation.

The maximum possible complexity of error generation is when combined errors from any number of qubits are generated. The $4^{K}-1$ error generators $F_{\alpha}$ for such a process are basis elements of $\mathfrak{g}=\mathfrak{s u}(n)=\mathfrak{s u}\left(2^{K}\right)$ in the defining ( $n$-dimensional) representation. As the defining representation is irreducible, there are no DF subspaces. We have written (17) in a notation compatible with the generalization to arbitrary Lie algebras done in Section IV] and more completely in [22].

\section{B. Irreducible Representations Have No Decoherence-Free Subsystems}

Knill, Laflamme, and Viola 11] discovered a method for decoherence-free coding into subsystems instead of into subspaces which has since received much attention; see [1, 14, 24]. In this section, we remark that in the situation of Assumption [1 existence of a DF subsystem implies that the representation is reducible.

The definition of a DF subsystem begins with the obvious statement that if the $F_{\alpha}$ are Hermitian and one of them is the identity, then the associative algebra $\mathcal{A}$ they generate is unital and closed under adjoints. Therefore, $C^{*}$-algebra methods may be applied.

Let $\mathcal{M}(d, \mathbb{C})$ denote the space of $d \times d$ complex matrices. Any basis for $\mathcal{H}$ determines a matrix representation $\phi: \mathcal{A} \rightarrow \mathcal{M}(d, \mathbb{C})$, where $d=\operatorname{dim}(\mathcal{H})$, simply by expressing each operator in this basis. The idea is that $\phi$ may be repetitive, i.e. it may happen that

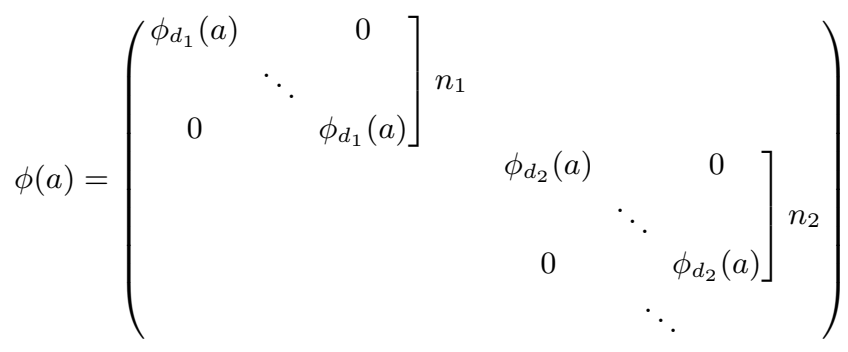

where $\phi_{d_{i}}: \mathcal{A} \rightarrow \mathcal{M}\left(d_{i}, \mathbb{C}\right)$. The $n_{1}, n_{2}, \ldots$ label the sizes of the corresponding sub-matrices. In fancier notation, $\phi(a)=\sum_{i} \mathbf{1}_{n_{i}} \otimes \phi_{d_{i}}(a)$. This is also called the central decomposition.

If this happens, then we may take a vector $\Psi \in \mathcal{H}$ which is also repetitive, so that the first $d_{1}$ components of $\Psi$ take the form $\alpha v$, where $\alpha \in \mathbb{C}, v \in \mathbb{C}^{d_{1}}$, and the second $d_{1}$ components take the form $\beta v$ for the same $v$, etc. We may do this $n_{1}$ times. Then $\phi(a) \Psi=(\alpha w, \beta w, \ldots)$ where $w=\phi_{d_{1}}(a) v$. The coefficient vector $(\alpha, \beta, \ldots) \in$ $\mathbb{C}^{n_{1}}$ can just as well be extracted after applying the operator $\phi(a)$ as before. In other words, the information 
contained in the $\alpha, \beta, \ldots$ is protected under this decoherence process.

Theorem 1. Decoherence-free subsystems do not exist if the error operators $F_{\alpha}$ generate an irreducible representation.

Proof. If the $F_{\alpha}$ generate an irreducible matrix representation of a semisimple Lie algebra $\mathfrak{g}$, then $\mathcal{A}$ will be the full algebra of Hermitian operators over $\mathcal{H}$. Therefore, the homomorphism $\phi: \mathcal{A} \rightarrow \mathcal{M}(d, \mathbb{C})$, where $d=\operatorname{dim}(\mathcal{H})$ is not repetitive. In the above notation, $n_{1}=1$, there is no $n_{2}$ and $d_{1}=\operatorname{dim}(\mathcal{H})$. Second proof: noiseless degrees of freedom are associated with observables in $\mathcal{A}^{\prime}$, the commutant algebra. By Schur's Lemma, $\mathcal{A}^{\prime}$ is the trivial algebra if $\phi$ is irreducible.

This theorem implies the corresponding result for DF subspaces as a special case. Conditions under which DF subspaces can exist have previously been studied in both the Lindblad (Markovian) formulation [8, 9] and for the non-Markovian case [6]. Theorem 11 extends their results to noiseless subsystems.

\section{Irreducible Representations Return to Equilibrium}

In this section we prove a property of the timeevolution of open quantum systems defined by irreducible representations. This property is not used directly in the rest of the paper, but it holds independent interest. The thermal Gibbs state

$$
\rho_{\beta}=\frac{e^{-\beta H}}{\operatorname{tr} e^{-\beta H}}
$$

is a stationary state for the Markovian dynamics of a system coupled to a heat bath. The question then arises: under what conditions does the system return to equilibrium for an initial state $\rho$ ?

Theorem 2. Suppose that a Markovian system coupled to a heat bath is described by Lindbladian (2) -(3), with Foperators that form a set of generators for a nontrivial ndimensional representation of a semisimple Lie algebra. The system returns to thermal equilibrium if and only if the representation is irreducible.

Proof. Return to equilibrium for arbitrary initial state $\rho$ will happen iff

$$
\lim _{t \rightarrow \infty} e^{\boldsymbol{L} t} \rho=\rho_{\beta} \text { for all } \rho \in \mathcal{D}(\mathcal{H}),
$$

where $\boldsymbol{L}=-i / \hbar\left[H_{A}, \rho\right]+\mathcal{L}(\rho)$ denotes the right side of (1), and $\mathcal{D}(\mathcal{H})$ denotes the space of density operators on $\mathcal{H}$. By assumption, the $F$-operators are Hermitian, in which case (8) is equivalent to the statement that any operator $X$ commuting with all the $V_{a}$ must look like $X=c \mathbf{1}$ for some constant $c$. This is equivalent by Schur's lemma to the statement that the $V_{a}$ generate an irreducible representation.

\section{Models of Decoherence}

Lidar et al. 9] classified the subspaces in which there is generically no decoherence in this model, where "generic" means that the decoherence is suppressed independently of the noise parameters (i.e. the $a$ matrix of (2) ) and of the initial conditions. If $\mathfrak{g}$ is semisimple (this assumption includes the Lie algebras of $S U(n), S O(n), S p(2 n), G_{2}, F_{4}, E_{6}, E_{7}, E_{8}$, and all direct sums of such algebras), then Lidar et al. 9] characterize the decoherence-free (DF) subspaces as those which are annihilated by every one of the representation matrices. At an intuitive level, this is not surprising since on such a subspace, the Lindblad operator $L_{F_{\alpha}, F_{\beta}}(\rho)=\left[F_{\alpha}, \rho F_{\beta}^{\dagger}\right]+\left[F_{\alpha} \rho, F_{\beta}^{\dagger}\right]$ is identically zero, and hence time-evolution is governed by the von Neumann equation. As representations of $\mathfrak{g}$, DF subspaces are always composed of singlets.

As discussed above, there are many interesting quantum systems which do not possess decoherence-free subspaces. Therefore, it is of interest to search for other effects which suppress decoherence to complement the existing techniques. On the other hand, at the highest level of generality, completely arbitrary decoherence is allowed by quantum mechanics. Thus it seems necessary to exploit symmetry in some way in order to achieve a suppression. Therefore we search for Lie-algebraic conditions which suppress the Lindbladian, but allowing some dependence on the noise parameters and on initial conditions.

The noise parameters and initial conditions, while difficult to experimentally fine-tune, are also not entirely out of the hands of laboratory control. Further, because the solution operator $e^{\boldsymbol{L} t}$ depends smoothly on any linear parameters contained in $\boldsymbol{L}$, the Lindbladian $\mathcal{L}(\rho)$ depends smoothly on the parameters $a_{\alpha, \beta}$, and hence a tiny variation in $a_{\alpha, \beta}$ results in a correspondingly small change in the dynamics.

\section{ALGEBRAIC IDENTITIES FOR LINDBLAD OPERATORS}

\section{A. Suppression of Decoherence Without Noiseless Subsystems}

Before continuing, we record a set of simple algebraic identities for Lindblad operators. The Lindblad operator (3) may be studied generally in terms of its matrix-valued bilinear form

$$
L_{A, B}=2 A \rho B^{\dagger}-\left\{\rho, B^{\dagger} A\right\} .
$$

Suppose that $A, B$ are both Hermitian. We assert that

$$
\begin{aligned}
& L_{A, B}=2[A, B] \rho+2 A[\rho, B]-B[\rho, A]-[\rho, B] A \\
& L_{A, B}+L_{B, A}=[A,[\rho, B]]+[B,[\rho, A]] \\
& L_{A, A}=[A,[\rho, A]] .
\end{aligned}
$$


hence if $A$ commutes with $\rho$ then $L_{A, A}=0$. If $[\rho, B]$ commutes with $A$ then

$$
L_{A, B}=2[A, B] \rho+A[\rho, B]-B[\rho, A] .
$$

The latter formula is antisymmetric in $A, B$. If $[\rho, A]$ also commutes with $B$ then (13) holds with $A$ and $B$ switched, hence $L_{A, B}=-L_{B, A}$. This also follows immediately from (11). If $[\rho, A]=A$ and $[\rho, B]=B$ then

$$
L_{A, B}=2[A, B](\rho+\mathbf{1}) \text {, hence } L_{A, B}=-L_{B, A} .
$$

We now investigate the consequences of Assumption 1 We identify elements $x \in \mathfrak{g}$ with the operators $\phi(x)$ acting on $\mathcal{H}$.

Lemma 1. Suppose that for each $x \in \mathfrak{g},[\rho, x]$ commutes with all of $\mathfrak{g}$ and $a_{\alpha \beta}$ is real. Then $\mathcal{L}(\rho)=0$.

Proof. The assumption entails that $\left[\rho, F_{\alpha}\right]$ commutes with $F_{\beta}$ for all $\alpha, \beta$. Then (11) implies that $L_{F_{\alpha}, F_{\beta}}=$ $-L_{F_{\beta}, F_{\alpha}}$ for all $\alpha, \beta$, but the sum $\mathcal{L}(\rho)$ involves these objects in symmetric combinations.

It is natural to ask: when can the conditions of Lemma 11 be satisfied? If $\rho$ can be expressed as a linear combination of elements of $\mathfrak{g}$ then the condition reduces to

$$
\operatorname{ad}_{\rho}(\mathfrak{g}) \subset Z(\mathfrak{g})
$$

where $Z(\mathfrak{g})$ is the center of $\mathfrak{g}$.

Generally, it may not be possible to express $\rho$ as a linear combination of (the identity and) generators of $\mathfrak{g}$ 22]. The vector space dimension $\operatorname{dim}(\mathfrak{g})$ will often be smaller than $n^{2}-1$, the geometric dimension of the space of density operators. However, it is possible to equip $\mathfrak{g}$ with an embedding into a larger algebra $\mathfrak{h}$, and note that the conditions of Lemma 1 will be satisfied if $\operatorname{ad}_{\rho}(\mathfrak{g})$ is contained in the centralizer of $\mathfrak{g}$ within $\mathfrak{h}$, i.e. the set of all elements in $\mathfrak{h}$ that commute with all elements in $\mathfrak{g}$.

Lemma 2. Suppose that $\operatorname{ad}_{\rho}$ is the identity on $\mathfrak{g}$ (i.e. $\left[\rho, F_{\alpha}\right]=F_{\alpha}$ for all $\alpha$ ) and $a_{\alpha \beta}$ is real. Then $\mathcal{L}(\rho)=0$.

Lemmas 11 and 2 point to the following general principle: suppression of decoherence, for an initial state $\rho$, is related to the adjoint action of the density matrix $\rho$ on the Lie algebra $\mathfrak{g}$ containing the error generators.

\section{B. Lindblad Operators for $\mathbf{s u}_{n}$}

The above analysis leads to exactly calculable formulae for Lindblad operators in the $\boldsymbol{n}$ representation of $\mathfrak{s u}(n)$. Let $\left\{X_{j}\right\}$ be the canonical set of generators for $\mathfrak{s u}(n)$ in this representation, satisfying $\operatorname{Tr}\left(X_{i} X_{j}\right)=2 \delta_{i j}$. MacFarlane et al. 25] give a very clear discussion of $\mathfrak{s u}(n)$ generators and the identities that they satisfy; we use the same notation and conventions.
The $L_{F_{\alpha}, F_{\beta}}$ are sums of commutators, hence they are traceless and may therefore be expressed as a linear combination of the $X_{i}$. An arbitrary $n \times n$ density matrix $\boldsymbol{\rho}_{v}$ may be written in the Bloch representation

$$
\boldsymbol{\rho}_{v}=\frac{1}{n}\left(\mathbf{1}+\sum_{a} v_{a} X_{a}\right), \quad v \in \mathbb{R}^{n^{2}-1}
$$

and write $L_{i j}$ for $L_{X_{i}, X_{j}}$.

For calculating with Lie algebras, we find it extremely useful to use a convention even lazier than the Einstein summation convention to systematically not write sums and repeated indices, as follows. Each $X_{a}$ is a matrix, but we never write the matrix indices; $v_{a}$ is a vector with the same dimension so $v \cdot X=\sum_{a} v_{a} X_{a}$ is a matrix. Extend this to all tensors, so that $v \cdot f_{b} \cdot X \longrightarrow \sum_{a, c} v_{a} f_{a b c} X_{c}$ etc.

The standard basis for the $\mathfrak{s u}(n)$ algebra satisfies the basic identities, which are derived in 25]:

$$
\begin{gathered}
{\left[\boldsymbol{\rho}_{v}, X_{b}\right]=\frac{2 i}{n} v \cdot f_{b} \cdot X, \quad X_{a} X_{b}=\frac{2}{n} \delta_{a b} \mathbf{1}+Q_{a b} \cdot X,} \\
{\left[X_{a}, X_{b}\right]=2 i f_{a b} \cdot X}
\end{gathered}
$$

This makes explicit calculations easy. For example, if the terms in the diagonal standard form (4) happen to be the $\mathfrak{s u}(n)$ generators, then using (12) we find

$$
L_{i i}=\left[X_{i}, \frac{2 i}{n} v \cdot f_{i} \cdot X\right]=\frac{4}{n} v \cdot f_{i} \cdot f_{i} \cdot X .
$$

Using [25, eq. (2.12)], we then have

$$
\sum_{i} L_{i i}=-4(v \cdot X)=4(\mathbf{1}-n \rho) .
$$

Thus we have derived a very simple formula for the Lindbladian of $\mathfrak{s u}(n)$-decoherence. It vanishes iff $\rho=\frac{1}{n} \mathbf{1}$.

\section{SYMMETRY BREAKING}

Symmetry breaking in this context can be modeled as the introduction of new error generators which do not belong to the representation of $\mathfrak{g}$ which gave the original symmetry. The standard assumption is that the symmetry is broken perturbatively by modification of the system-bath Hamiltonian:

$$
H_{A B} \rightarrow H_{A B}+\epsilon H_{I, \mathrm{sb}} .
$$

where $H_{I \text {,sb }}$ denotes a symmetry-breaking interaction between the system and the bath, and $\epsilon \ll 1$. As discussed by Lidar et al. 9], the new terms added to the Lindbladian are of the form

$$
\mathcal{L}^{\prime}(\rho)=\sum_{\alpha, p}\left(\widetilde{a}_{\alpha p} L_{F_{\alpha}, \epsilon G_{p}}+\text { h.c. }\right)+\sum_{p, q} \widetilde{b}_{p q} L_{\epsilon G_{p}, \epsilon G_{q}}
$$

where h.c. denotes the hermitian conjugate. Clearly the terms proportional to $L_{\epsilon G_{p}, \epsilon G_{q}}$ are $O\left(\epsilon^{2}\right)$ and can be neglected in first-order perturbation theory, so we turn to the analysis of the $O(\epsilon)$ terms in the present context. 
Definition 1. Let $\mathfrak{h}$ be a Lie algebra with subspaces $\mathfrak{g}, \mathfrak{f}$. (a) Following standard textbooks, we define the centralizer of $\mathfrak{g}$ in $\mathfrak{h}$ by

$$
Z_{\mathfrak{h}}(\mathfrak{g})=\{x \in \mathfrak{h}:[x, g]=0 \forall g \in \mathfrak{g}\} .
$$

(b) Let $\phi: \mathfrak{h} \rightarrow \operatorname{End}(V)$ be a representation of $\mathfrak{h}$ on a vector space $V$ and let $\rho$ be an operator on $V$. We say that a Lie algebra homomorphism $A: \mathfrak{h} \rightarrow \mathfrak{h}$ switches centralizers for $\mathfrak{g}, \mathfrak{f}$ if

$$
A(\mathfrak{f}) \subset Z_{\mathfrak{h}}(\mathfrak{g}) \text { and } A(\mathfrak{g}) \subset Z_{\mathfrak{h}}(\mathfrak{f}) \text {. }
$$

We also say that $\rho$ switches centralizers if $A=\operatorname{ad}_{\rho}$ does. ${ }^{1}$

Assume that $\mathfrak{h}$ admits a faithful representation $\phi: \mathfrak{h} \rightarrow$ $\mathfrak{g l}(\mathcal{H})$ on the system Hilbert space. Since $\phi$ is faithful, identify $\mathfrak{h}$ with its image under $\phi$, which makes sense since we are just interested in operators on $\mathcal{H}$.

Let $\mathfrak{f}$ and $\mathfrak{g}$ be the Lie algebras generated by the $F$ operators and the $G$-operators respectively. If $\rho$ switches centralizers for $\mathfrak{f}$ and $\mathfrak{g}$, then (11) implies

$$
L_{F_{\alpha}, \epsilon G_{p}}=-L_{\epsilon G_{p}, F_{\alpha}}
$$

and therefore, the corresponding term in (17) is proportional to

$$
\widetilde{a}_{\alpha p}-\widetilde{a}_{\alpha p}^{*}=2 i \operatorname{Im}\left(a_{\alpha p}\right) .
$$

If the couplings $\widetilde{a}_{\alpha p}$ are real, then the $O(\epsilon)$ symmetry breaking terms (17) are zero, and the symmetry is perturbatively stable (to first order). We have proven:

Theorem 3. Let $\mathfrak{f}$ and $\mathfrak{g}$ be the Lie algebras generated by the $F$-operators and the $G$-operators respectively. If $\rho$ switches centralizers for $\mathfrak{f}$ and $\mathfrak{g}$, and $\widetilde{a}_{\alpha p} \in \mathbb{R}$ for all $\alpha, p$, then $\mathcal{L}^{\prime}(\rho)=0$. If $\widetilde{a}_{\alpha, p}$ has an imaginary part, then the $O(\epsilon)$ terms in $\mathcal{L}^{\prime}(\rho)$ are proportional to $\operatorname{Im}\left(\widetilde{a}_{\alpha, p}\right)$.

Physically, these interactions are safe for quantum computing, because they do not contribute to decoherence. It is therefore of interest to locate examples of density matrices which switch centralizers for certain subalgebras.

The most obvious example is the following. Suppose that $\mathfrak{h}=\mathfrak{f} \oplus \mathfrak{g}$ is a direct sum, so in an appropriate basis we can write all of the representation matrices in Block diagonal form; schematically,

$$
\phi(\mathfrak{h})=\left(\begin{array}{cc}
\phi(\mathfrak{f}) & 0 \\
0 & \phi(\mathfrak{g})
\end{array}\right) .
$$

We will now continue with our convention of identifying $\mathfrak{f}$ with its image under the faithful representation $\phi$. Suppose that $\rho$ is also Block diagonal in the same basis, so that $[\rho, \mathfrak{f}] \subset \mathfrak{f}$ and $[\rho, \mathfrak{g}] \subset \mathfrak{g}$. Under these conditions, the identity homomorphism switches centralizers, i.e. $\mathfrak{f} \subset Z_{\mathfrak{h}}(\mathfrak{g})$ and $\mathfrak{g} \subset Z_{\mathfrak{h}}(\mathfrak{f})$. Therefore, $\operatorname{ad}_{\rho}$ also

\footnotetext{
${ }^{1}$ While Def. (a) is standard, I have never seen Def. \b) before.
}

switches centralizers. This, like any example with a DF subsystem, is a reducible representation, but it is plausible that centralizer-switching occurs for irreducible representations as well.

It is fruitful to apply this analysis to the case of a symmetry-breaking perturbation (17). Theorem 3 implies that if $\mathfrak{f}$ and $\mathfrak{g}$ switch centralizers, then the crossterms, which are those proportional to $\epsilon L_{F_{\alpha}, G_{p}}$ in (17), are zero. These are also the only $O(\epsilon)$ terms, so this kind of symmetry breaking is a purely $O\left(\epsilon^{2}\right)$ effect!

\section{THE EFFECT OF COARSE-GRAINING}

\section{A. Lie Algebra Channels}

Quantum channels defined by Lie algebra representations were defined and studied extensively in a separate paper 22]. Here we recall the basic definitions and set notation, before discussing the relationship to Markovian dynamics.

Definition 2. Let $\mathfrak{g}$ denote a Lie algebra of dimension $k$, and let $\left\{X_{i}: i=1, \ldots, k\right\}$ be a basis of $\mathfrak{g}$. Let $\phi$ be a unitary representation of $\mathfrak{g}$ on a Hilbert space $\mathcal{H}$. The generalized depolarizing channel or Lie algebra channel is defined to be the channel in which an error occurs conditionally with probability $p$, causing an initial state $|\psi\rangle \in \mathcal{H}$ to evolve into an ensemble of the $k$ states $\phi\left(X_{i}\right)|\psi\rangle$, all with equal likelihood.

The Kraus operators for the channel of Definition 2 are given by

$$
M_{0}=\sqrt{1-p} 1, \quad M_{i}=\sqrt{\frac{p}{Z}} \phi\left(X_{i}\right) .
$$

where $Z$ is a normalization constant, and will be determined momentarily. The operators $M_{\mu}$ are hermitian if the representation is unitary and if $p \in[0,1]$.

Let $K$ be the Killing form. If $\phi$ is irreducible and the chosen basis satisfies pseudo-orthonormality

$$
K\left(X_{i}, X_{j}\right)=\mathfrak{n} \delta_{i j}, \quad \mathfrak{n} \neq 0,
$$

then $\sum_{i} \phi\left(X_{i}\right)^{2}=Z \cdot \mathbf{1}$ with $Z=\mathfrak{n} c_{2}(\phi)$ where $c_{2}(\phi)$ is the quadratic Casimir, and therefore the condition of probability conservation $M_{\mu} M^{\mu}=\mathbf{1}$ is satisfied. If $\phi$ is irreducible but the basis does not satisfy (19), then Def. 2 does not define a channel. If $\phi$ is reducible, then one must replace $Z \rightarrow \sum_{p} Z_{p}$ where the $Z_{p}$ are the normalization constants for the independent irreducible components.

Using (18), the Lie algebra channel has the explicit Kraus decomposition

$$
\boldsymbol{\rho} \rightarrow \mathcal{E}(\boldsymbol{\rho})=(1-p) \boldsymbol{\rho}+\frac{p}{Z} \sum_{i=1}^{k} \phi\left(X_{i}\right) \boldsymbol{\rho} \phi\left(X_{i}\right) .
$$

In what follows, we will write $X_{i}$ for $\phi\left(X_{i}\right)$ since the distinction is clear from context. 
The companion paper [22] gave methods for calculating with these channels using Lie algebra identities. Let $\mathcal{E}_{\mathfrak{g}}$ denote the channel based on the fundamental representation of $\mathfrak{g}$; for example, $\mathcal{E}_{\mathfrak{s u}(n)}$ is based on the $\boldsymbol{n}$ of $\mathfrak{s u}(n)$. The action of $\mathcal{E}_{\mathfrak{s u}(n)}$ on a general hermitian matrix $\boldsymbol{\rho}=n^{-1}(\operatorname{tr}(\boldsymbol{\rho}) \mathbf{1}+v \cdot X)$ can be calculated exactly in closed form, yielding

$$
\mathcal{E}\left(\boldsymbol{\rho}_{v}\right)=\frac{1}{n}\left(\operatorname{tr}(\boldsymbol{\rho}) \mathbf{1}+\frac{(1-p) n^{2}-1}{n^{2}-1} v \cdot X\right) .
$$

In the qubit case, the coefficient of $v$ is $1-4 p / 3$, which is exactly consistent with the standard qubit depolarizing channel (6)-(7).

\section{B. Coarse-graining}

Markovian dynamics may be derived from quantum theory of measurement by coarse-graining time. Expanding Lindblad's equation $\dot{\rho}=-\frac{1}{2} \sum_{\alpha} a_{\alpha}\left(\left[F_{\alpha}, \rho F_{\alpha}^{\dagger}\right]+\right.$ $\left.\left[F_{\alpha} \rho, F_{\alpha}^{\dagger}\right]\right)$ to first order in the short time interval $\tau$ yields

$$
\rho(t+\tau)=M_{0} \rho(t) M_{0}+\tau \sum_{\alpha} F_{\alpha} \rho(t) F_{\alpha}^{\dagger},
$$

with

$$
M_{0}=\mathbf{1}-\frac{\tau}{2} \sum_{i} F_{i}^{\dagger} F_{i}
$$

Suppose that the Lindblad operators equal to properly normalized Lie algebra generators, $F_{i}=Z^{-1 / 2} X_{i}$. Then

$$
M_{0}=\left(1-\frac{\tau}{2}\right) \mathbf{1} \sim \sqrt{1-\tau} \mathbf{1}+O\left(\tau^{2}\right)
$$

Also defining

$$
M_{i}=\sqrt{\frac{\tau}{Z}} X_{i}
$$

shows that the coarse-grained Lindblad's equation is completely equivalent to the Lie algebra channel (18) to first order in $\tau$, under the mapping $\tau \rightarrow p$.

It is interesting that in the Lie algebra channel, $p$ is probability of error while in Markovian dynamics, $\tau$ is the coarse-graining time. In fact, their equivalence to first order coincides with one of the defining properties of a Poisson process: that the probability of exactly one change in a sufficiently small time interval of length $\tau$ is proportional to $\tau$, with constant of proportionality given by the event rate for the generation of errors. This assumption is often made (either implicitly or explicitly) in the theory of quantum error correction and describes a quantum Poisson process. An example of a quantum Poisson process is spontaneous emission [26]. For such processes, we expect $p \propto \tau$ to first order in $p$ or $\tau$.

If we specialize to $\mathfrak{g}=\mathfrak{s u}(n)$, then the evolution of the channel was found to be (21) with $p$ replaced by $\tau$. Since (21) is already linear in $p$, any $O\left(\tau^{2}\right)$ terms just come along for the ride. The conclusion is that in $\mathfrak{s u}(n)$ Markovian dynamics,

$$
\rho(t+\tau) \sim \frac{\operatorname{tr} \rho(t)}{n} \mathbf{1}+\frac{1}{n}\left(1-\frac{\tau n^{2}}{n^{2}-1}\right) v(t) \cdot X
$$

Eqn. (22) is something which, a priori we did not expect: an explicit formula, valid to first order in the coarsegraining time, for the nontrivial Markovian system defined by $\mathfrak{s u}_{n}$. The simplification here is related to the simplification (15) of the Lindbladian.

\section{CONCLUSIONS}

Since there are many examples in which no decoherence-free subsystems exist (among them all cases where the error generators act irreducibly on the system Hilbert space), it is of interest to search for novel mechanisms which suppress decoherence in these more general cases. We presented three results (Lemmas 1 and 2 Theorem 3) which indicate decoherence suppression without the need for noiseless subsystems. There is a certain trade-off; our methods do not necessarily apply to an arbitrary initial density matrix, or for completely generic noise parameters $a_{\alpha \beta}$. On the other hand, our computational methods are novel and the result-suppression of decoherence in the error-algebra approach without noiseless subsystems-is an interesting new direction which warrants further study.
[1] M. A. Nielsen and I. L. Chuang, Quantum computation and quantum information (Cambridge University Press, Cambridge, 2000).

[2] C. H. Bennett and P. W. Shor, IEEE Trans. Inform. Theory 44, 2724 (1998), information theory: 1948-1998.

[3] D. Gottesman, Phys. Rev. A (3) 54, 1862 (1996).

[4] E. Knill and R. Laflamme, Phys. Rev. A (3) 55, 900 (1997).

[5] A. R. Calderbank, E. M. Rains, P. W. Shor, and N. J. A. Sloane, Phys. Rev. Lett. 78, 405 (1997).
[6] P. Zanardi and M. Rasetti, Modern Phys. Lett. B 11, 1085 (1997).

[7] P. Zanardi and M. Rasetti, Phys. Rev. Lett. 79, 3306 (1997), quant-ph/9705044.

[8] P. Zanardi, Phys. Rev. A57, 3276 (1998), quantph/9705045.

[9] D. A. Lidar, I. L. Chuang, and K. B. Whaley, Phys. Rev. Lett. 81, 2594 (1998), quant-ph/9807004.

[10] D. A. Lidar, D. Bacon, and K. B. Whaley, Phys. Rev. Lett. 82, 4556 (1999), quant-ph/9809081. 
[11] E. Knill, R. Laflamme, and L. Viola, Phys. Rev. Lett. 84, 2525 (2000).

[12] L. Viola and S. Lloyd, Phys. Rev. A (3) 58, 2733 (1998).

[13] M. H. Freedman, A. Kitaev, M. J. Larsen, and Z. Wang, Bull. Amer. Math. Soc. (N.S.) 40, 31 (2003), mathematical challenges of the 21st century (Los Angeles, CA, 2000).

[14] P. Zanardi, Phys. Rev. A (3) 63, 012301 (2001).

[15] M. Rasetti and P. Zanardi, in Proceedings of the Adriatico Conference on Quantum Interferometry, III (Trieste, 1999) (2000), vol. 48, pp. 559-572.

[16] P. Zanardi and S. Lloyd, Phys. Rev. Lett. 90, 067902 (2003), quant-ph/0208132.

[17] H.-P. Breuer and F. Petruccione, The theory of open quantum systems (Oxford University Press, New York, 2002).

[18] G. Lindblad, Comm. Math. Phys. 48, 119 (1976).

[19] F. Benatti and R. Floreanini, eds., Irreversible Quantum
Dynamics, vol. 622 of Lecture Notes in Physics (SpringerVerlag, Berlin, 2003).

[20] D. Bacon, D. A. Lidar, and K. B. Whaley, Phys. Rev. A60, 1944 (1999), quant-ph/9902041.

[21] R. Alicki and K. Lendi, Quantum dynamical semigroups and applications, vol. 286 of Lecture Notes in Physics (Springer-Verlag, Berlin, 1987).

[22] W. G. Ritter, J. Math. Phys. (submitted) (2005), quant$\mathrm{ph} / 0502153$.

[23] H. Georgi, Lie algebras in particle physics, vol. 54 of Frontiers in Physics (Benjamin/Cummings Publishing Co. Inc., Reading, Mass., 1982).

[24] S. De Filippo, Phys. Rev. A (3) 62, 052307 (2000).

[25] A. J. MacFarlane, A. Sudbery, and P. H. Weiz, Commun. Math. Phys. 11, 77 (1968).

[26] C. Ahn, H. M. Wiseman, and G. J. Milburn, Phys. Rev. A 67, 052310 (2003). 